\title{
Study on the Different Method of Extraction of Star Anise Oil
}

\author{
Cheng Haidi ${ }^{1, a^{*}}$, Sun Tianmin ${ }^{1, b}$ \\ ${ }^{1}$ School of Chemistry and Bioengineering, Qilu Insititute of Technology, Jinan, Shandong, 250200, China
}

\begin{abstract}
The three reagents of anhydrous ethanol, petroleum ether and ethyl acetate were selected by soxlet extraction, steam distillation and ultrasonic extraction to extract the star anise oil from the fine anise powder. Three kinds of methods for extracting star anise oil were studied on different pattern organic solvent. The result of the different volume fractions of ethanol is that the highest extraction rate is $99.7 \%$ ethanol. It can be seen that the extraction rate are: ethanol $>$ petroleum ether $>$ acetic acid ethyl ester. The different methods of extracting the star anise oil shows that the extraction rate are: soxhlet extraction method $>$ ultrasonic extraction method $>$ steam distillation extraction method. In summary, it can be seen by soxhlet extraction method and the best solvent is $99.7 \%$ ethanol.
\end{abstract}

\section{Introduction}

Star anise is also known as star anise and anise. Its trees grow in southwest China with an annual output of 3000 tons. Its color is dark brown and has a sweet taste and a strong aromatic smell, which comes from the volatile anisaldehyde in it. Dried and mature anise contains about $5 \%-8 \%$ aromatic oil, about $22 \%$ fatty oil, protein and shikimic acid, etc ${ }^{[1-2]}$. Star anise oil is a kind of natural flavor oil with complex components extracted from the fruits or leaves of anise, generally as a volatile oil-like liquid. The essential oil mainly contains anisole, in addition, it also contains anisaldehyde, p-allyl isoprene, flavonoids and organic acids ${ }^{[3-4]}$.

In the past decades, star anise is just a condiment to the cook. With the development of science and technology, more and more active components of star anise had been discovered. In recent years, star anise oil has been proved to have a benefit on bacteriostatic, insecticidal and anti-free radical oxidation activities. The main ingredient in star anise oil, anisole, was also found that exhibits various pharmacological properties such as anesthetic, antimicrobial, anti-inflammatory and antioxidant activities $^{[5-6]}$. Besides, anisole has been conveniently transformed to a functional monomer which possess good thermostability and good mechanical properties ${ }^{[7-10]}$.

In the traditional use, star anise fruit is directly used, and the utilization rate of star anise oil is low. With the development of the food industry, the extraction of star anise oil has replaced the use of star anise fruit because of its small size, sanitation, easily to use, and less added amount. In order to improve the yield of star anise oil, it is necessary to study the extraction technology of star anise oil.

At present, the common extraction methods of anisole oil at home and abroad include soxhlet extraction, steam distillation, ultrasonic extraction, supercritical $\mathrm{CO}_{2}$ fluid extraction, etc ${ }^{[1-13]}$. Different extracted solvent not only has a great influence on the work of star anise oil and its components, but also its physical properties ${ }^{[14-}$ 16].

There are few studies on the influence of content determination and extraction effect under laboratory conditions. The methods adopted in this study are soxhlet extraction, steam distillation and ultrasonic extraction to extract star anise oil. The differences between the same method and different solvent extraction and the differences between different methods to extract star anise oil were studied.

\section{MATERIALS AND METHODS}

\subsection{Materials and measurements}

All commercially available chemicals were of reagent grade and used as received without further purification. The star anise powder can be obtained by drying the star anise under $100^{\circ} \mathrm{C}$ for more than $4 \mathrm{~h}$, grinding and sifting through a suitable sieve. Infrared spectra were measured on a Nicolet 380 FT-IR spectrometer in the region of $400 \sim 4000 \mathrm{~cm}-1$ using KBr pellets.

\footnotetext{
* Corresponding author: achenghaidi163@126.com

b1172171458@qq.com
} 


\subsection{Method of extraction}

\subsubsection{Soxhlet extraction}

The device diagram is shown in the figure 1.

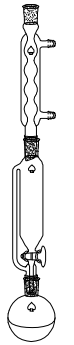

Figure 1 Soxhlet extraction

Extraction by different solvents A mixture of $18.0 \mathrm{~g}$ star anise powder and $80 \mathrm{~mL}$ organic solvent (ethanol/petroleum ether/ethyl acetate) was stirred under $50 \mathrm{~V}$. When mixture is close to colorless, stop heating. Vacuum distillation with rotary evaporator will steam out a lot of solvent. The distillation ends when no condensation drops. After cooling, the mass of the product was weighed and the extraction rate was calculated.

Extraction by different volume fractions of ethanol A mixture of $18.0 \mathrm{~g}$ star anise powder and $80 \mathrm{~mL}$ ethanol was stirred under $50 \mathrm{~V}$ volts. When mixture is close to colorless, stop heating. Repeating the above operation and calculating the extraction rate was calculated.

\subsubsection{Water vapor distillaiton}

The device diagram is shown in the figure 2.

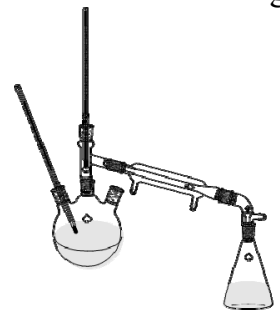

Figure 2 Water vapor distillation

A mixture of $30.0 \mathrm{~g}$ star anise powder and $150 \mathrm{~mL}$ distilled water was stirred and refluxed for 3 hours. When mixture is nearly colorless, stop heating. After distillating and cooling, the mass of the product was weighed and the extraction rate was calculated.

\subsubsection{Ultrasonic extraction}

A mixture of $10.0 \mathrm{~g}$ star anise powder and $200 \mathrm{~mL}$ organic solvent(ethanol/petroleum ether/ethyl acetate) was carried by ultrasonic cleaner under the temperature of $40^{\circ} \mathrm{C}$ for one hour. After distillating and cooling, the mass of the product was weighed and the extraction rate was calculated.

\section{Results \& Discussion}

\subsection{FTIR measurement results}

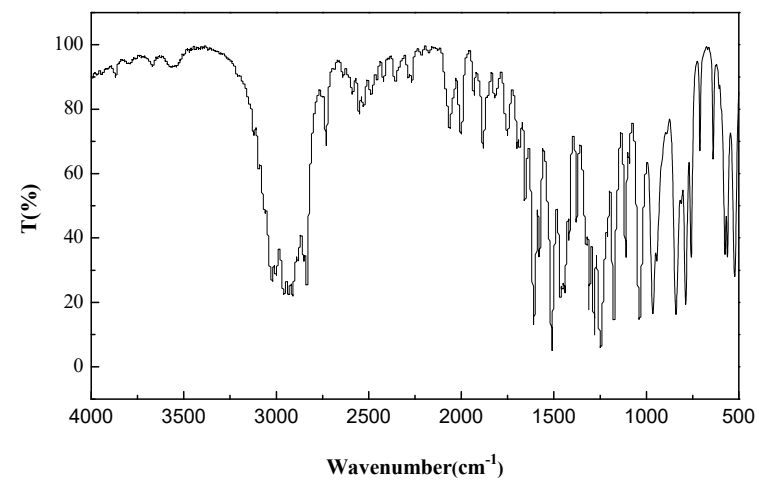

Figure 3 The infrared spectrum of star anise oil

Since star anise oil contains $80-90 \%$ of anisole, infrared spectrum is used to determine whether the extract contains anisole. As shown in figure 3: $3005.73 \mathrm{~cm}-1$ (C-H); $2934.65 \mathrm{~cm}-1$ (-CH3); $2856.72 \mathrm{~cm}-1$ $(-\mathrm{CH} 2) ; \quad 1600.50 \mathrm{~cm}-1 \quad(\mathrm{C}=\mathrm{C}) ; 1458.34 \mathrm{~cm}-1 \quad(-\mathrm{CH} 2)$; $1000 \mathrm{~cm}-1(\mathrm{C}-\mathrm{O})$ and $843.55 \mathrm{~cm}-1(\mathrm{C}-\mathrm{H}$ on the benzene ring). It can be seen that the extraction of star anise contains various functional groups such as double bond, alkoxy groups, benzene ring in the molecule, which is basically consistent with the known structure.

\subsection{Different organic solvent by soxhlet extraction}

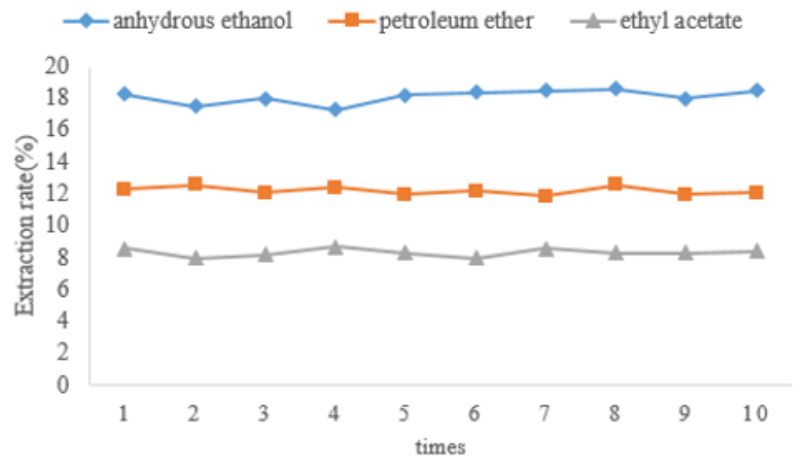

Figure 4 Extraction rates under different solvents In the soxhlet method, anhydrous ethanol, petroleum ether and ethyl acetate were used to extract aniseed oil under the same experimental conditions. As can be seen from the figure 4, when anhydrous ethanol was used as an organic solvent, the extraction rate of star anise oil was the highest, with the highest value being $18.6 \%$. 


\subsection{Different volume fractions of ethanol by soxhlet extraction}

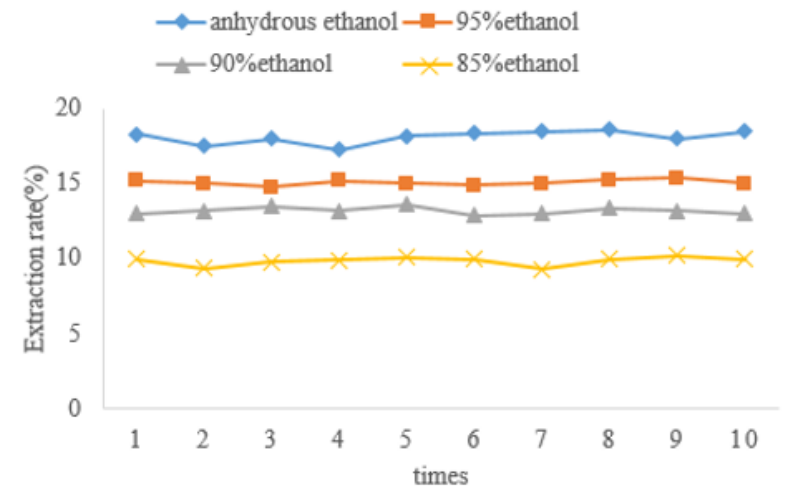

Figure 5 Extraction rates under different volume fractions of ethanol

In order to observe whether the concentration of ethanol has any effect on the extraction rate of star anise oil, experiments were carried out at the volume fractions of ethanol of $85 \%, 90 \%, 95 \%$ and $99.7 \%$ (anhydrous ethanol) respectively. As can be seen from the figure 5, the highest extraction rate was $99.7 \%$ of anhydrous ethanol.

\subsection{Different organic solvent by ultrasonic extraction}

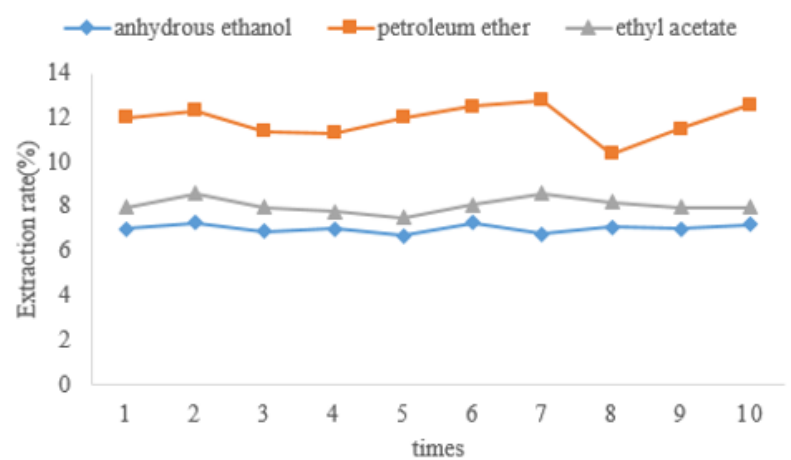

Figure 6 Extraction rates under different organic solvent by ultrasonic extraction

Anhydrous ethanol, petroleum ether and ethyl acetate were used to extract star anise oil under the same experimental conditions by ultrasonic cleaner. As can be seen from the figure, when petroleum ether was used as an organic solvent, the extraction rate of star anise oil was the highest, with the highest value being $12.8 \%$.

\subsection{Comparison of three methods}

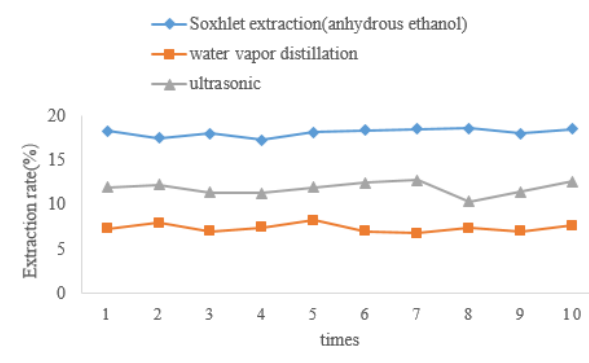

Figure 7 Extraction rates under comparison of three methods
Compared with the three methods, it can be seen from figure that the extraction yield of soxhlet extraction is significantly higher than the other two methods.

\section{Conclusions}

The result of the different volume fractions of ethanol is that the highest extraction rate is $99.7 \%$ ethanol. It can be seen that the extraction yield are: ethanol $>$ petroleum ether $>$ acetic acid ethyl ester. Extracting the star anise oil from different methods shows that the extraction yield are: soxhlet extraction method $>$ ultrasonic extraction method $>$ steam distillation extraction method. In summary, it can be seen by soxhlet extraction method and the best solvent is $99.7 \%$ ethanol.

\section{Acknowledgments}

This work was financially supported by the research foundation of Qilu Institute of Technology.

\section{References}

1. Davis M C, Guenthner A J, Sahagun C M, et al. Polymer, 54(26), 6902-6909, (2003)

2. Hua-Wei, Yuan, et al.China Condiment, (2018)

3. Dong-Mei, H. E, et al. Food Research and Development, (2009)

4. Elmhalli F, Katinka Pålsson, Jan Örberg, et al. Experimental and Applied Acarology, (2018)

5. Fengkai He, Kaikai Jin, Yuanqiang Wang, et al. ACS Sustainable Chemistry \& Engineering, (2017)

6. Tao, YQ, FK, et al. Polymer Chemistry, 8(13), 2010-2015, (2017)

7. Ya-Ting L I, Ling L I, Yi Z, et al. ence and Technology of Food Industry, (2019)

8. Rudzki E, Grzywa Z. Contact Dermatitis, 2010, 2(6), 305-308, (2019)

9. Cai M, Guo X , Liang $\mathrm{H}$, et al. International Journal of Food ence \& Technology, 48(11), 23242330, (2013)

10. Cai M, Guo X, Liang H, et al. International Journal of Food ence \& Technology, 48(11), 2324-2330, (2013)

11. Qi L, Yaoyuan Z, He N I, et al. Journal of South China Normal University(Natural ence Edition), (2019)

12. Jun-Jie G, Xian-Fang M, Qian W. Education Modernization, (2019)

13. Yong, Guo, M. Jian-Hua, L. Yan-Guo. Natural Product Research \& Development, (2008)

14. Gang, Chen , G. Xiao-Lei . Modern Food ence \& Technology, (2010)

15. Matsushita, Takaya, et al. Journal of the ence of food and agriculture, (2018) 
16. Ping LI, Zhan S, Xiaoxia S, et al. Food science, (2017) 